\title{
Cardiac Sarcoidosis or Giant Cell Myocarditis? On Treatment Improvement of Fulminant Myocarditis as Demonstrated by Cardiovascular Magnetic Resonance Imaging
}

\author{
Hari Bogabathina, ${ }^{1}$ Peter Olson, ${ }^{1}$ Vikas K. Rathi, ${ }^{2}$ and Robert W. W. Biederman' \\ ${ }^{1}$ Department of Cardiovascular Magnetic Resonance Imaging, Gerald McGinnis Cardiovascular Institute, Allegheny General Hospital, \\ 320 East North Avenue, Pittsburgh, PA 15212, USA \\ ${ }^{2}$ Department of Cardiovascular Magnetic Resonance, Bon Secours Heart \& Vascular Institute, 5949 Harbour Park Drive, \\ Midlothian, VA, USA
}

Correspondence should be addressed to Robert W. W. Biederman, Rbiederm@wpahs.org

Received 28 September 2011; Accepted 17 October 2011

Academic Editors: M. Barros, K. N. Blackett, M.-R. Chen, J. Peteiro, and H. Ueda

Copyright (C) 2012 Hari Bogabathina et al. This is an open access article distributed under the Creative Commons Attribution License, which permits unrestricted use, distribution, and reproduction in any medium, provided the original work is properly cited.

Giant cell myocarditis, but not cardiac sarcoidosis, is known to cause fulminant myocarditis resulting in severe heart failure. However, giant cell myocarditis and cardiac sarcoidosis are pathologically similar, and attempts at pathological differentiation between the two remain difficult. We are presenting a case of fulminant myocarditis that has pathological features suggestive of cardiac sarcoidosis, but clinically mimicking giant cell myocarditis. This patient was treated with cyclosporine and prednisone and recovered well. This case we believe challenges our current understanding of these intertwined conditions. By obtaining a sense of severity of cardiac involvement via delayed hyperenhancement of cardiac magnetic resonance imaging, we were more inclined to treat this patient as giant cell myocarditis with cyclosporine. This resulted in excellent improvement of patient's cardiac function as shown by delayed hyperenhancement images, early perfusion images, and SSFP videos.

\section{Introduction}

Fulminant myocarditis is an extremely severe form of heart failure, with short duration of onset, requiring the use of inotropic support and often cardiac mechanical assistive support. Giant cell myocarditis (GCM) is a well-noted cause of fulminant myocarditis [1]. Infiltrative cardiomyopathies, including idiopathic granulomatous myocarditis and cardiac sarcoidosis (CS), typically run a chronic course and are not known to cause fulminant myocarditis [1]. Recently, fulminant myocarditis has been designated a Class 1 indication for endomyocardial biopsy (EMB), as patients with definitive pathological diagnosis and appropriate treatment have good prognosis [1].

There is an ongoing debate whether GCM is a distinct pathological entity from CS or GCM is a part of the spectrum of pathology in CS [2]. The multicenter observational study by Okura et al. has given some insight into the pathological and clinical distinctions between GCM and CS. GCM pathologically has giant cells, eosinophils, lymphocytic inflammatory infiltrate, and prominent myocyte necrosis $[2,3]$. CS on the other hand has noncaseating granulomatous inflammation, predominant fibrosis, and without prominent myocyte necrosis [2]. Notably, both GCM and CS had equivalent numbers of giant cells [2]. CS had a more indolent clinical course, with more likelihood of bradyarrhythmias, and better prognosis [2].

Until recently, it has been difficult to diagnose CS premortem. Endomyocardial biopsy although definitive when positive has a poor negative predictive value secondary to skip lesions [4]. Recently, DHE-CMR has shown promise in reliably diagnosing CS with excellent sensitivity and specificity. In our lab, we diagnose CS definitively by utilizing DHE-CMR [5]. Of the 81 patients with biopsy-proven 


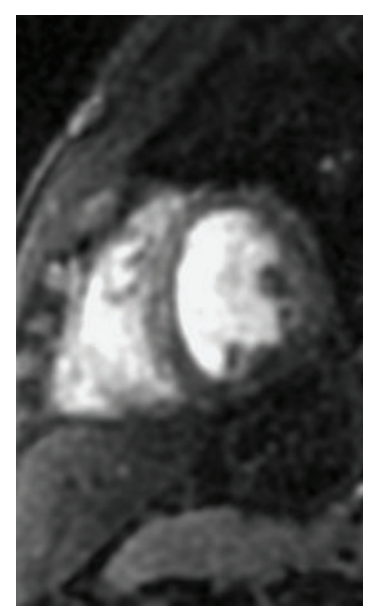

(a)

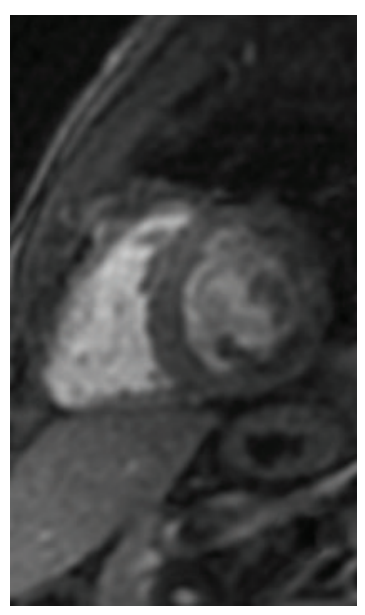

(b)

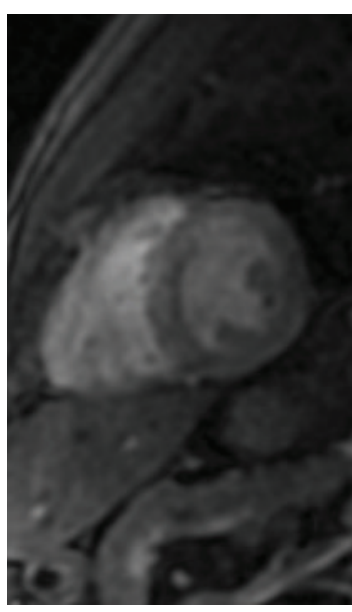

(c)

Figure 1: First-pass perfusion images. Subendocardial perfusion defects seen on these first-pass perfusion images in the initial exam when patient was critically ill (a) do not conform to any coronary distribution and have improved in the study done in seven weeks on cyclosporine and prednisone (b), and show stabilization from then on as evidenced on the study done in nine months (c).

extracardiac sarcoidosis 21 patients (26\%) had CS by DHECMR [6]. DHE-CMR demonstrated sensitivity, specificity, positive predictive value, negative predictive value, and overall accuracy of $100 \%, 78 \%, 55 \%, 100 \%$, and $83 \%$, respectively, in the diagnosis of CS among patients with pulmonary sarcoidosis [7]. To our knowledge, there has only been two case reports of GCM diagnosed by CMR $[8,9]$. We describe a case of fulminant myocarditis, which has pathological features of CS, clinically mimicking GCM, treated as GCM based on the extensive myocardial involvement on CMR.

\section{Case Presentation}

A 42-year-old African-American male presented to our hospital with a 2-week history of rapidly progressive exertional dyspnea. He had no significant past medical history, did not take any medications, had no allergies, occasional cigarette smoking, drank an average of six-pack of beer a week, and his parents had hypertension and diabetes.

Cardiac enzymes were negative, BNP level was 843 , and D-dimer was elevated at 3.7. A urine drug screen, HIV 1/HIV 2 antibodies, and hepatitis viral panel were negative. Rheumatoid factor was normal, ANA titer was mildly positive with a speckled pattern, angiotensin converting enzyme level was high at $70 \mathrm{U} / \mathrm{L}$ (normal 7 to 46), histoplasma antigen was negative, parvovirus B-19 IgM antibody was negative, and parvovirus IgG antibody was positive suggesting a past infection. Patient's EKG showed sinus rhythm, low voltage, and poor R-waves in inferior and anterior leads. CXR showed pulmonary edema and cardiomegaly, with nodular pattern in the periphery. CT chest with contrast showed no evidence of pulmonary embolism, significant degree of mediastinal, hilar, right peridiaphragmatic lymphadenopathy, small pleural effusion, small pericardial effusion, and bilateral multifocal consolidation.

TTE showed severe global hypokinesis with regional variation, mostly sparing the apex.
Tc-99 tetrofosmin with adenosine stress testing showed a medium to large, severe, fixed anterior perfusion defect with sparing of the apex, suggesting an anterior infarct, although the distribution was atypical for a proximal LAD occlusion. Severe global LV systolic dysfunction was also noted.

Left and right heart catheterization showed normal coronary arteries, elevated right atrial pressures, moderate pulmonary hypertension, elevated pulmonary capillary wedge pressure, elevated LVEDP, decreased cardiac output and index, no intra- or extracardiac shunts were noted. Patient was anticoagulated and placed on intra-aortic balloon pump for afterload reduction.

Cardiovascular magnetic resonance imaging (CMR) was done for further evaluation of fulminant myocarditis. CMR showed top normal left ventricular size, mildly dilated by $3 \mathrm{D}$, LVEF of $27 \%$, with severe regional wall dysfunction, worse at base and sparing the apex (see Video 1 in Supplementary Material available on line at doi:10.1155/2011/647041). Firstpass perfusion demonstrated nonspecific subtle subendocardial hypoperfusion defect, not following any coronary distribution (Figure 1). Delayed hyperenhancement imaging (DHE) showed an extremely heterogeneous, dense, and patchy, near complete enhancement of the myocardium, with increased T1 signal by gadolinium imaging. Late imaging after the postedema washout period reveals a marked patchy signal consistent with a severe inflammatory or infiltrative process (Figure 2). Right ventricular segmental dysfunction exactly colocalized with the transmural RV signal. Right paratracheal and perihilar lymphadenopathy was also noted. The official CMR report suggested that this pattern was most consistent with GCM.

Based on the CMR findings and the clinical presentation EMB was done. The EMB showed noncaseating granulomatous inflammation with many multinucleated giant cells, histiocytes, and mild chronic inflammation (Figures 3 and 4). Special stains were negative for organisms. No viral cytopathic effect was seen. Iron stain was negative. 


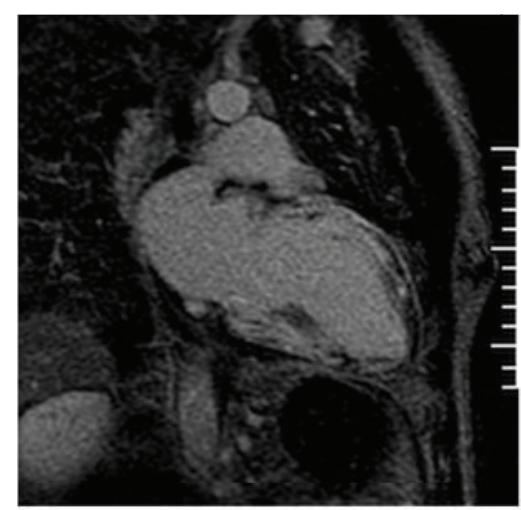

(a1)

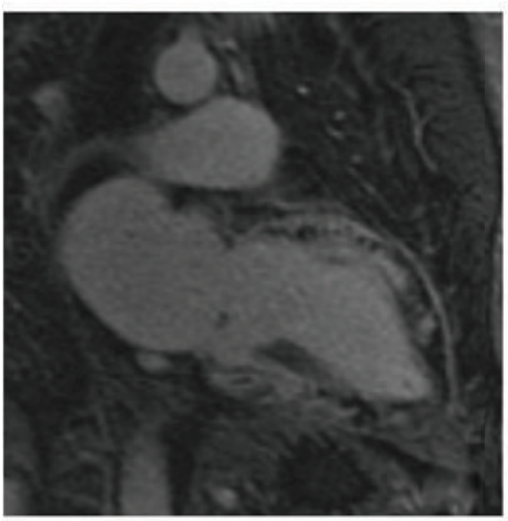

(b1)

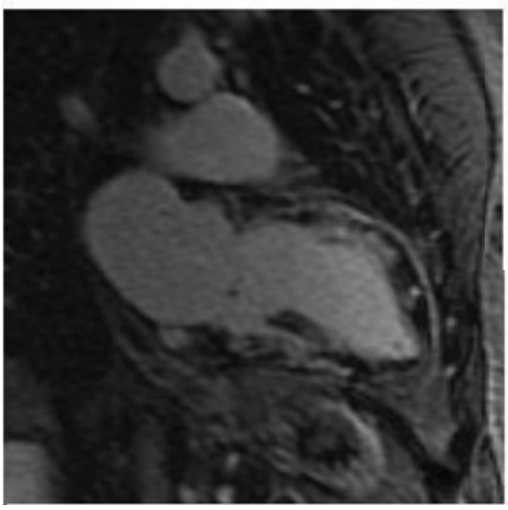

(c1)

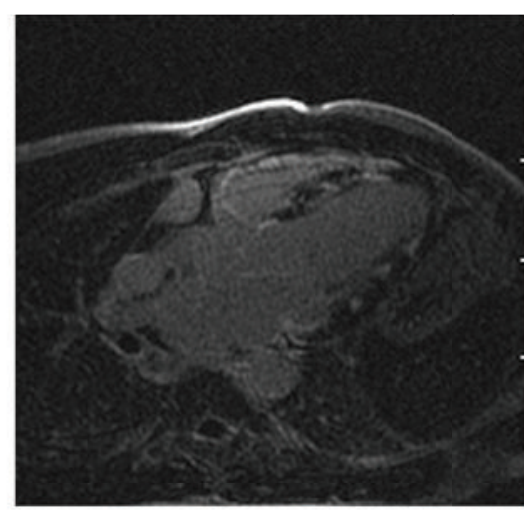

(a2)

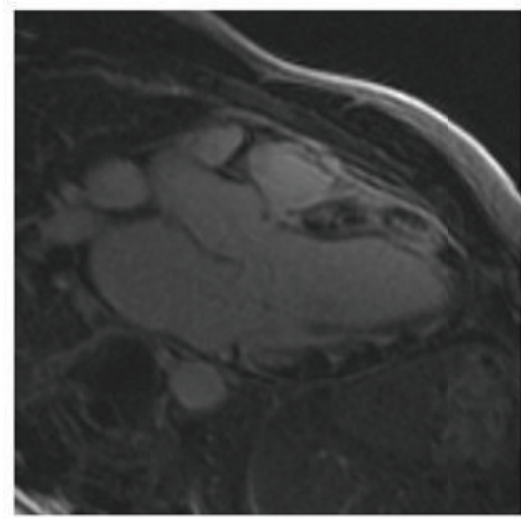

(b2)

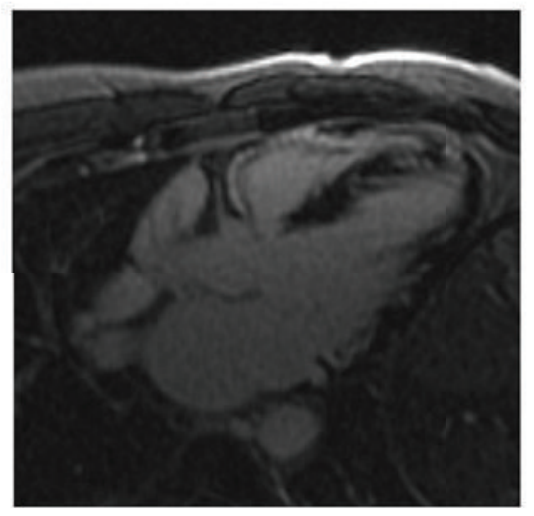

(c2)

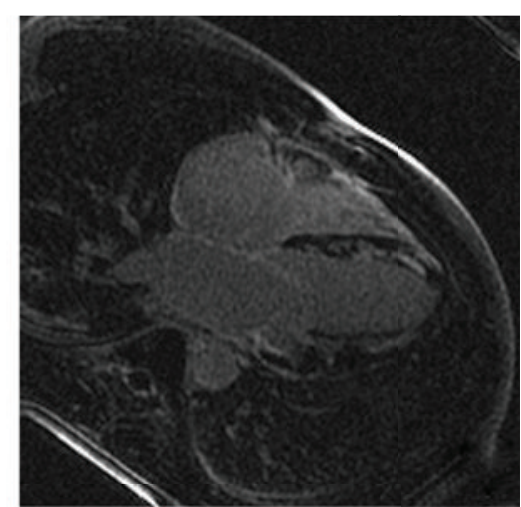

(a3)

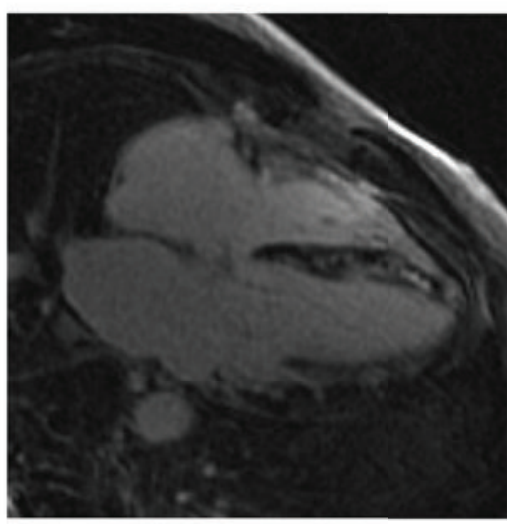

(b3)

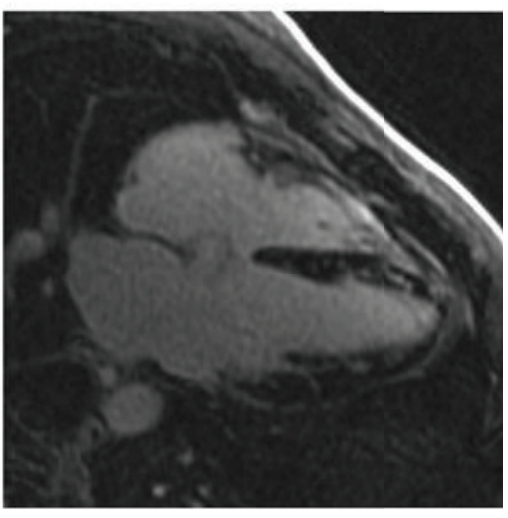

(c3)

FIGURE 2: Delayed gadolinium hyperenhancement images. DHE-CMR pattern of patchy delayed hyperenhancement secondary to giant cell myocarditis or a severe form of cardiac sarcoidosis. Patchy hyperenhancement in left ventricular wall and diffuse enhancement in right ventricular wall in the initial exam (a1, a2, and a3) has improved to coalescence of hyperenhancement in seven weeks on cyclosporine and prednisone (b1, b2, and b3). The nine-month exam (c1, c2, and c3) shows a stabilization of change in hyperenhancement as compared to the seven-week exam.

Thioflavin-T stain and congo red stain for amyloid were negative. Sarcoidosis/granulomatous myocarditis was favored over idiopathic giant cell myocarditis because of the presence of well-formed granulomas and granulomatous inflammation. Interestingly, the first official pathology read was signed out as GCM. Thus, the patient was initiated on prednisone and cyclosporine $50 \mathrm{mg}$ PO BID which was later increased to $75 \mathrm{mg}$ PO BID in 6 days, in specific treatment of GCM.
With rapid clinical improvement from $\mathrm{CHF}$, the IABP was removed. After clinical stabilization, the patient was discharged home on carvedilol, aspirin, lisinopril, prednisone slow taper over 5 weeks, cyclosporine at $75 \mathrm{mg}$ PO BID, and Bactrim SS for prophylaxis while on cyclosporine.

Repeat CMR 7 weeks after the initial CMR showed improved LVEF to $40 \%$ (Video 2). The first-pass perfusion imaging showed hypoperfused subendocardial defects 


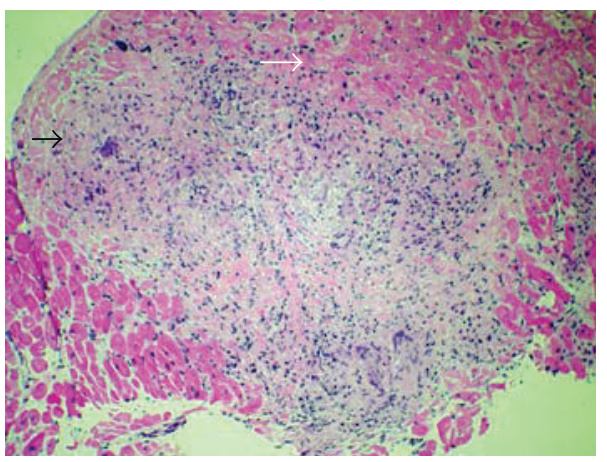

Figure 3: Hematoxylin and Eosin staining of myocardial biopsy specimen at 100x resolution demonstrating well-formed granuloma (black arrow) and myocardial fascicles (white arrow).

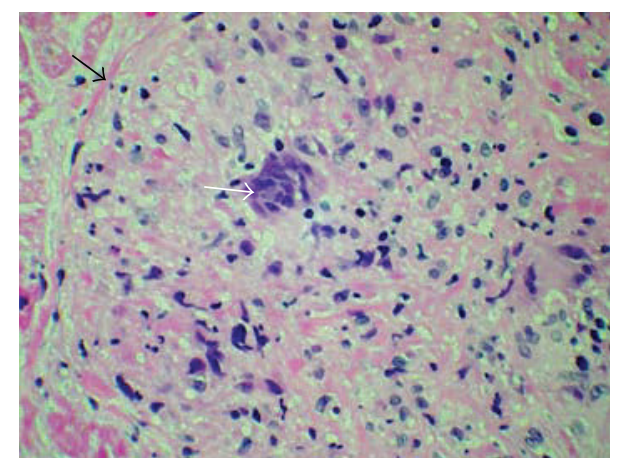

Figure 4: Hematoxylin and Eosin staining of myocardial biopsy specimen at 200x resolution demonstrating well-formed granuloma (black arrow) with a multinucleated giant cell in the center (white arrow).

remained generalized (Figure 1). DHE showed moderate improvement and coalescence of previously seen LV myocardial enhancement with mild to moderate improvement in RV signal as well (Figure 2). Also noted was the interval improvement in the mediastinal lymphadenopathy.

Repeat CMR 9 months later showed the LVEF stabilized at $40 \%$ and RVEF at $45 \%$ (Video 3). First-pass perfusion by gadolinium revealed an unchanged generalized subendocardial, near circumferential hypoperfusion defect (Figure 1). The DHE pattern had mildly improved as well (Figure 2). Also noted was the interval improvement of the pulmonary sarcoidosis pattern since the second CMR.

\section{Discussion}

Fulminant myocarditis has a limited differential diagnosis including GCM, necrotizing eosinophilic myocarditis, lymphocytic myocarditis, and acute myocardial infarction [1]. GCM is the leading offender among the nonischemic presentations. Although, after extensive work of gathering all available cases, clinical and pathological distinctions between GCM and CS were made by Okura et al., demonstrating a significant number of cases, there is significant overlap in both pathological and clinical findings [2].
Our patient presented with severe heart failure, which quickly deteriorated into fulminant heart failure, requiring IABP and inotropic support. An intrepid diagnosis of GCM was made on CMR. EMB showed giant cells and granulomatous inflammation, pointing to a diagnosis of GCM versus CS. However, the acute clinical presentation, the extensive nature of myocarditis, involving entire heart by CMR forced us to consider a diagnosis of GCM and overlap with CS, thus treating the patient with cyclosporine and steroids. Over the next 7 weeks, patient's EF improved from $27 \%$ to $40 \%$. Cyclosporine-based immunosuppression as treatment for GCM has been studied and showed good prognosis and prolonged transplant-free survival $[10,11]$. Steroids are the mainstay of treatment for CS, and only a few studies have looked at immunosuppressive agent use for treatment [12].

Serial CMRs over a nine month period demonstrated improving ejection fraction, decreasing inflammation, and coalescing of enhancement via DHE corresponding to the changing pattern of fibrosis that correlated with marked and durable clinical improvement.

From our review of literature, there has been one reported case of GCM imaged by CMR, but diagnosis was confirmed only on pathology of explanted heart. Ongoing controversy regarding partial overlap of GCM with CS suggests that immunomodulatory therapies are beneficial in both settings, such that astute clinical recognition and rapid institution of immunologic therapy are critical to patient prognosis.

\section{Conclusion}

The extent of cardiac involvement and improvement on immunomodulator and steroid treatment is well demonstrated by CMR imaging. Perfusion CMR imaging and DHECMR imaging can be used to monitor treatment change in myocardial pathology. This case sheds more light on CS and GCM being a spectrum of pathology rather than separate entities.

\section{Consent}

Verbal informed consent was obtained from the patient for publication of this case report and accompanying images. A copy of the verbal consent is available for review by the Editor-in-Chief of this journal.

\section{Conflict of Interests}

The authors declare that there is no conflict of interests.

\section{Author's Contributions}

H. Bogabathina prepared the manuscript. P. Olson was the pathologist for this case and obtained the pathology slides for this paper. V. K. Rathi was involved in imaging the patient utilizing CMR and contributed to the preparation of this paper. R. W. W. Biederman was involved in clinical cardiac care, imaged the patient utilizing CMR, and supervised 
the preparation of this manuscript. All authors contributed equally to this work.

\section{References}

[1] L. T. Cooper, K. L. Baughman, A. M. Feldman et al., "The role of endomyocardial biopsy in the management of cardiovascular disease: a scientific statement from the American Heart Association, the American College of Cardiology, and the European Society of Cardiology," Circulation, vol. 116, no. 19, pp. 2216-2233, 2007.

[2] Y. Okura, G. W. Dec, J. M. Hare et al., "A clinical and histopathologic comparison of cardiac sarcoidosis and idiopathic giant cell myocarditis," Journal of the American College of Cardiology, vol. 41, no. 2, pp. 322-328, 2003.

[3] M. J. Davies, A. Pomerance, and R. D. Teare, "Idiopathic giant cell myocarditis: a distinctive clinico pathological entity," British Heart Journal, vol. 37, no. 2, pp. 192-195, 1975.

[4] A. Uemura, S. I. Morimoto, S. Hiramitsu, Y. Kato, T. Ito, and H. Hishida, "Histologic diagnostic rate of cardiac sarcoidosis: evaluation of endomyocardial biopsies," American Heart Journal, vol. 138, no. 2 I, pp. 299-302, 1999.

[5] R. W. W. Biederman, M. Doyle, and J. Yamrozik, The Cardiovascular MRI Tutorial : Lectures and Learning, Lippincott Williams \& Wilkins, Philadelphia, Pa, USA, 2008.

[6] R. J. Kim, M. R. Patel, P. J. Cawley et al., "Detection of myocardial damage in patients with sarcoidosis," Circulation, vol. 120, no. 20, pp. 1969-1977, 2009.

[7] J. P. Smedema, G. Snoep, M. P. G. Van Kroonenburgh et al., "Evaluation of the accuracy of gadolinium-enhanced cardiovascular magnetic resonance in the diagnosis of cardiac sarcoidosis," Journal of the American College of Cardiology, vol. 45, no. 10, pp. 1683-1690, 2005.

[8] J. R. Shonk, J. Vogel-Claussen, M. K. Halushka, J. A. C. Lima, and D. A. Bluemke, "Giant cell myocarditis depicted by cardiac magnetic resonance imaging," Journal of Computer Assisted Tomography, vol. 29, no. 6, pp. 742-744, 2005.

[9] A. Azarine, R. Guillemain, and P. Bruneval, "Different focal delayed gadolinium-enhancement patterns using cardiac magnetic resonance in a case of diffuse giant cell myocarditis," European Heart Journal, vol. 30, no. 12, p. 1485, 2009.

[10] L. T. Cooper Jr., G. J. Berry, and R. Shabetai, "Idiopathic giant-cell myocarditis-natural history and treatment," New England Journal of Medicine, vol. 336, no. 26, pp. 1860-1866, 1997.

[11] L. T. Cooper, "Acute heart failure due to fulminant and giant cell myocarditis," Herz, vol. 31, no. 8, pp. 767-770, 2006.

[12] B. Pierre-Louis, A. Prasad, and W. H. Frishman, "Cardiac manifestations of sarcoidosis and therapeutic options," Cardiology in Review, vol. 17, no. 4, pp. 153-158, 2009. 


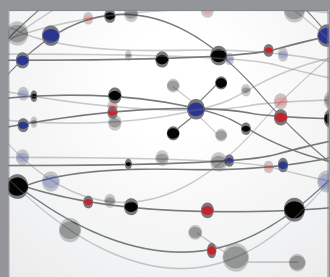

The Scientific World Journal
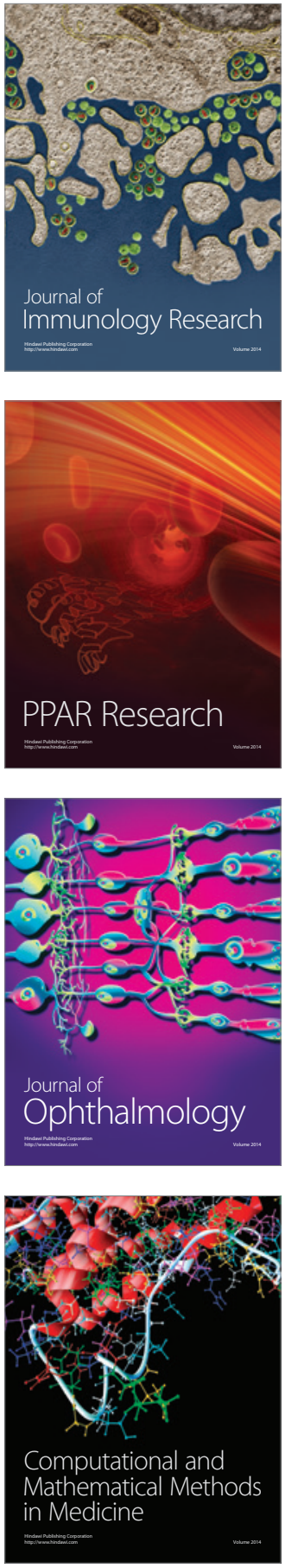

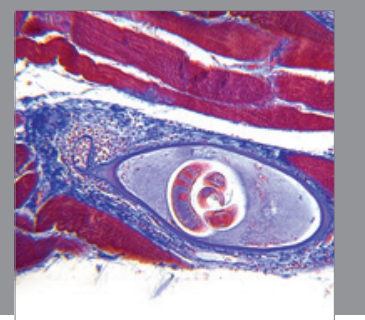

Gastroenterology

Research and Practice
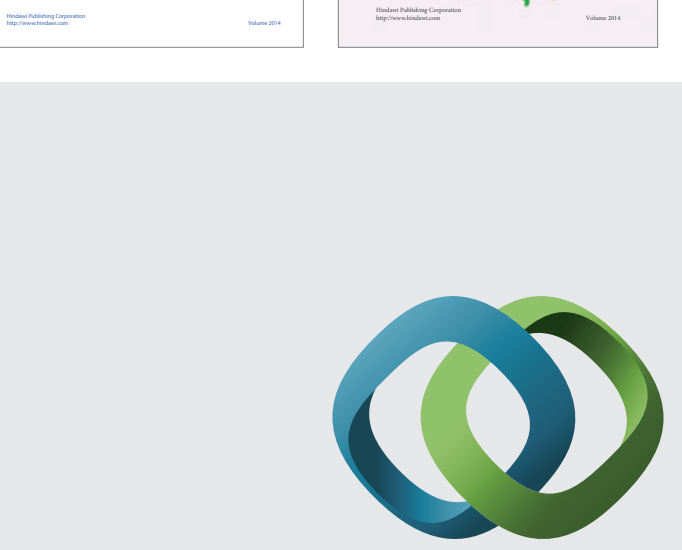

\section{Hindawi}

Submit your manuscripts at

http://www.hindawi.com
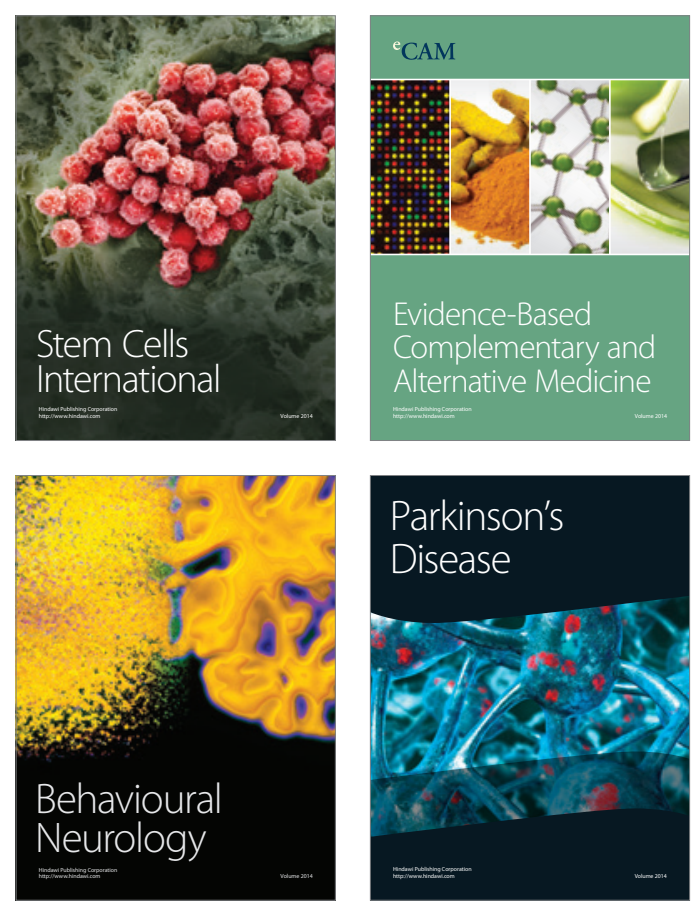

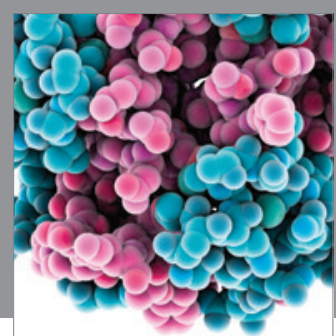

Journal of
Diabetes Research

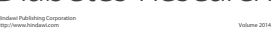

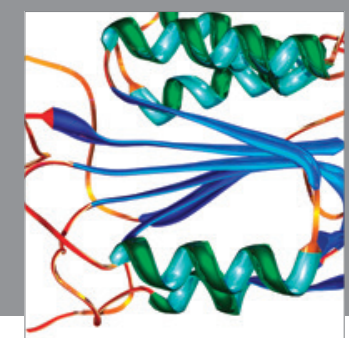

Disease Markers
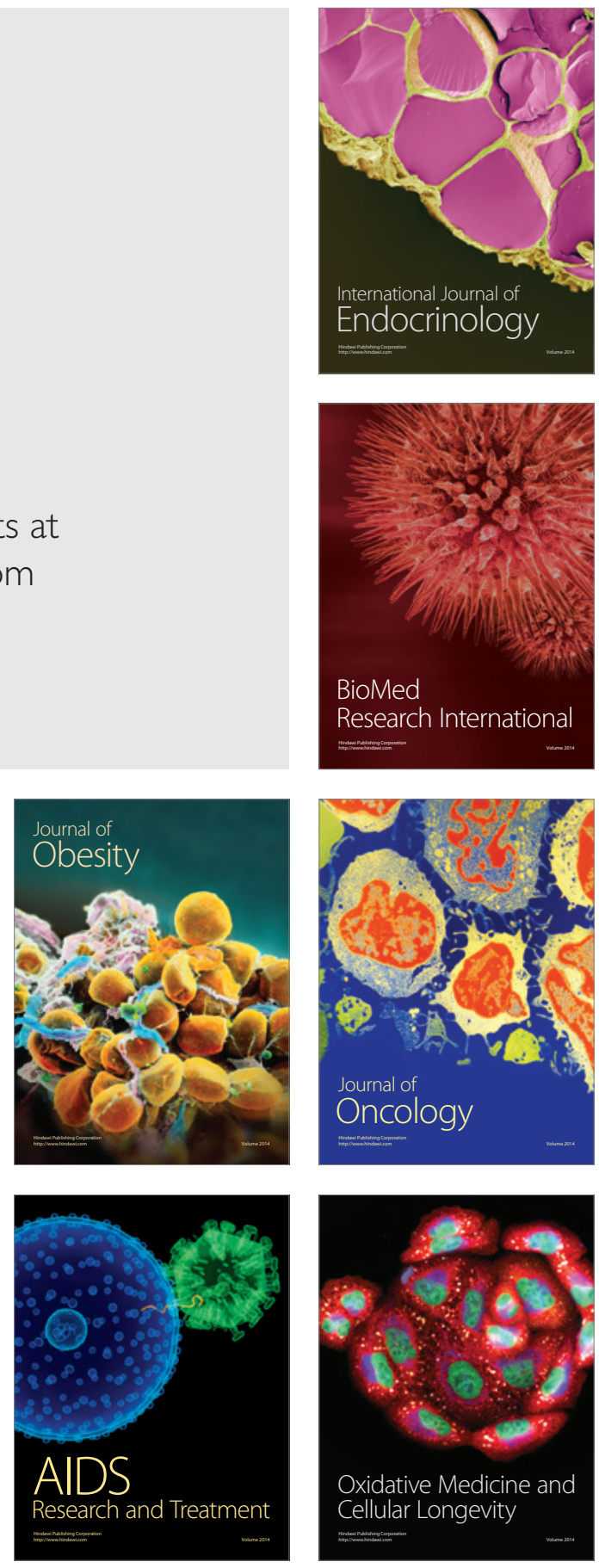